\title{
Management of the Aymand's Hernia in Laparoscopy
}

\author{
Radwan Kassira, c, Michel Bourbon ${ }^{\mathrm{b}}$
}

\begin{abstract}
A case of acute appendicitis within an inguinal hernia known as Amyand's hernia, is presented. Aymand's hernia is a very rare condition. Amyand's hernia is rarely diagnosed preoperatively. We report a very rare case of appendicitis presenting in an incarcerated inguinal hernia. It's a first case report of Aymand's hernia in laparoscopy. An 83-year-old woman was admitted to our emergency department with a painful groin mass. Ultrasonographic examination revealed a hernia sac containing suspected bowel segment. A diagnostic laparoscopy was performed initially and disclosed a inguinal hernia containing a strangulated appendix with a necrotic tip. Without inguinal incision, we were able to obtain a correct diagnosis and perform an appendectomy. This kind of hernia poses diagnostic and therapeutic dilemmas. Initial diagnostic laparoscopy is an invaluable adjunct in both diagnosis and treatment of incarcerated inguinal hernias. Aymand's hernia is a rare condition in which the Ultrasound proves to be a most useful tool. Before the final choice of treatment, general surgeons should bear in mind this rare presentation of acute appendicitis.
\end{abstract}

Keywords: Diagnostic laparoscopy; Amyand's hernia; Appendicitis; Inguinal hernia; Ultrasonography

\section{Introduction}

A case of acute appendicitis within an inguinal hernia known as Amyand's hernia, is presented. Aymand's hernia is a very

Manuscript accepted for publication August 14, 2013

${ }^{a}$ Department of Digestive Surgery, CHU Hospital, Jean Monnet University, Saint Etienne, France

${ }^{\mathrm{b}}$ Department of Digestive Surgery, clinique chirurgicale mutualiste, Saint Etienne, France

${ }^{\mathrm{c}}$ Corresponding author: Radwan Kassir, Department of Digestive Surgery, CHU Hospital, Jean Monnet University, Avenue Albert Raimond, 42270 Saint Etienne, France.

Email: Radwankassir42@hotmail.Fr

doi: http://dx.doi.org/10.4021/jcs194w rare condition. Amyand's hernia is rarely diagnosed preoperatively.

\section{Case Report}

We report the case of an 81-year-old woman who admitted to the emergency room of the surgical clinic due to a pain and swelling in the right inguinal region that had appeared suddenly 5 days before. Her medical history included hypertension, gastroesophageal reflux disease and carotid endarterectomy. She had no fever neither vomiting. Physical examination revealed a painful inguinal hernia and not reducible. Her laboratory findings were within normal. Sonographic examination of the right groin area showed a hernia sac containing suspected bowel segment (Fig. 1).

The presumed diagnosis was an inguinal hernia with bowel incarceration . Surgical groin exploration by laparoscopy revealed an inguinal hernia containing a strangulated appendix with a necrotic tip (Fig. 2, 3). The appendix was resected and the hernia was repaired (Fig. 4). Histology

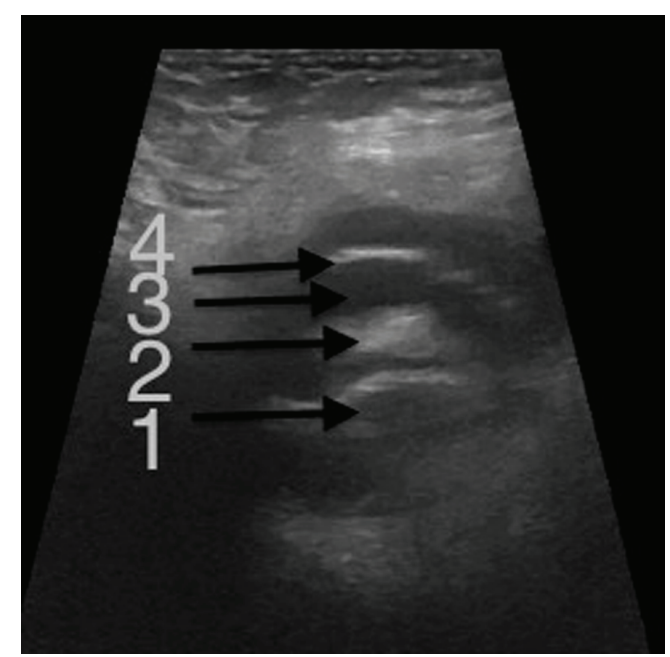

Figure 1. Transverse scan ( sonography) of the right groin showing the fluid-filled hernia sac containing the appendix. 1 = appendicitis; 2 = mesoappendix; 3 = effusion; $4=$ hernia sac. 


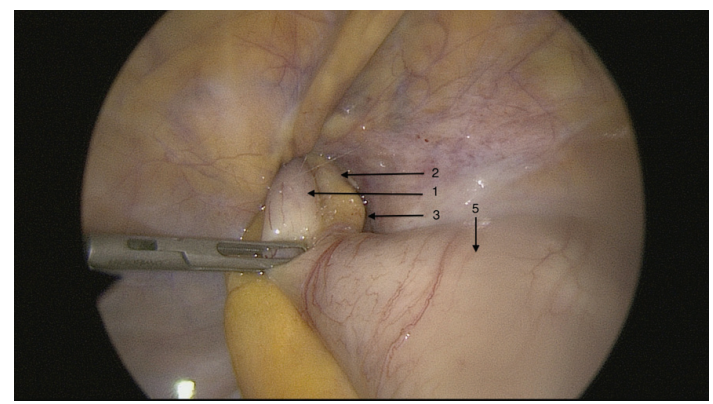

Figure 2. Laparoscopic view of the twisted and incarcerated appendix. 1 = appendicitis; 2 = mesoappendix; 3 = effusion; $5=$ caecum.

showed acute ischemic and suppurative appendicitis with perforation. The postoperative period was simple and the patient was discharged home 5 days later.

\section{Discussion}

The incidence of appendicitis within an inguinal hernia is low, estimated at $0.07 \%[1,2]$. The presence of an appendix within an inguinal hernia sac was first described by Claudius Amyand in 1735, hence the entity takes its name [3]. The first published case by Amyand was in London an 11-yearold boy complaining of right inguinal hernia . Inflammation of the appendix is attributed to external compression of the appendix at the neck of the hernia. The physiology of our case is still undetermined. Either the existence of the appendix in the hernia sac is the initial incident and strangulation of the appendix occurred, or the appendicitis started first and then the inflamed appendix moved into the hernia sac.

Historically Amyand's hernia is diagnosed intra-operatively after open exploration of the groin. We were able to obtain a correct diagnosis and perform an appendectomy without inguinal incision.

Even for the experienced surgeon, the hernias often pose technical dilemmas [4]. The surgeon may encounter unusual findings like Aymand's hernia. No standard approach to

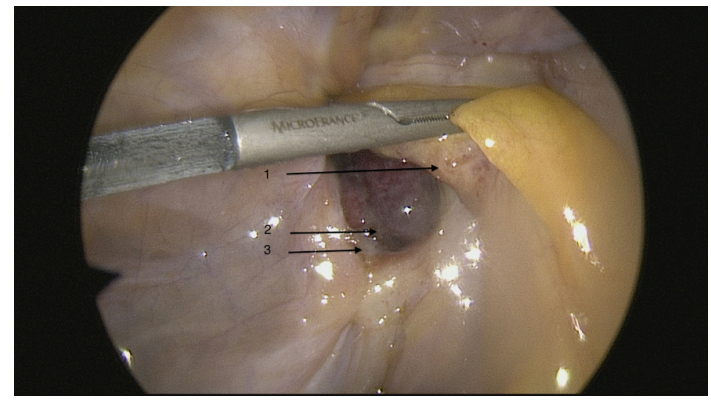

Figure 3. Laparoscopic view of the necrotic tip. 1 = appendicitis; 2 = the necrotic tip; 3 = effusion.

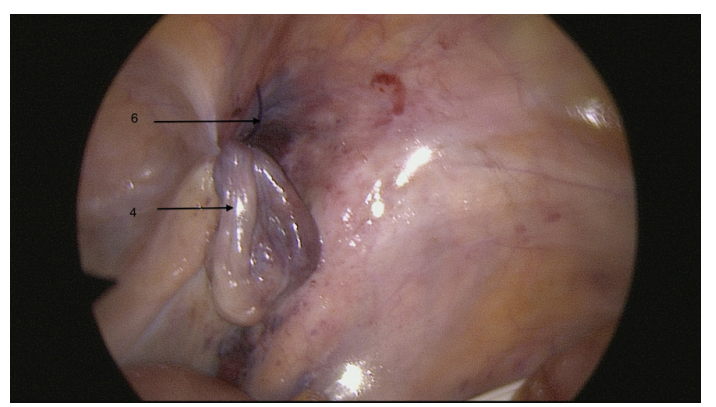

Figure 4. Laparoscopic view of the hernia orifice after repair. $4=$ hernia sac. $6=$ hernia was repaired by an endoloop.

treatment of this hernia has been described, possibly due to the rarity of this condition. Hernia repair should be without making use of plugs or synthetic meshes due to the high risk of suppuration of such materials [4-6].

Compared with open interventions, the advantages of laparoscopic hernia treatment like the shorter hospital stay and earlier return to work have been extensively studied. The use of both methods in the case of an incarcerated hernia is open to dispute. In our patient, the unusual presentation of the hernia prompted us to do a diagnostic laparoscopy first. The appendix was seen entering the hernia sac. Therefore we were able to obtain a correct diagnosis and perform an appendectomy before opening the sac, eliminating the need for open exploration and contamination of the groin.

Amyand's hernia is a rare entity which is hard to diagnose preoperatively. We wish to underline the usefulness of initial diagnostic laparoscopy in both the diagnosis and treatment of atypical hernias like Amyand's hernias.

\section{Conclusion}

Although the incidence of de Armyand's hernia is extremely low, surgeons should be aware of the existence of this kind of atypical presentation of appendicitis. Aymand's hernia is a rare condition in which the Ultrasound proves to be a most useful tool. Diagnostic laparoscopy could be a valuable tool in the correct diagnosis and management of unusual presentations of incarcerated groin hernias.

\section{Conflict of Interest}

The authors declare no conflict of interest.

\section{References}

1. Sharma H, Gupta A, Shekhawat NS, Memon B, Memon MA. Amyand's hernia: a report of 18 consecutive patients over a 15-year period. Hernia. 2007;11(1):31-35. 
2. D'Alia C, Lo Schiavo MG, Tonante A, Taranto F, Gagliano E, Bonanno L, Di Giuseppe G, et al. Amyand's hernia: case report and review of the literature. Hernia. 2003;7(2):89-91.

3. Milanchi S, Allins AD. Amyand's hernia: history, imaging, and management. Hernia. 2008;12(3):321-322.

4. Ballas K, Kontoulis T, Skouras C, Triantafyllou A, Symeonidis N, Pavlidis T, Marakis G, et al. Unusual findings in inguinal hernia surgery: report of 6 rare cases. Hippokratia. 2009;13(3):169-171.

5. Losanoff JE, Basson MD. Amyand hernia: a classification to improve management. Hernia. 2008;12(3):325326.

6. Livaditi E, Mavridis G, Christopoulos-Geroulanos G. Amyand's hernia in premature neonates: report of two cases. Hernia. 2007;11(6):547-549. 